\title{
Potential Predictors of Motor and Functional Outcomes After Distributed Constraint-Induced Therapy for Patients With Stroke
}

\author{
Keh-chung Lin, ScD, OTR, Yan-hua Huang, PhD, OTR, Yu-wei Hsieh, MS, and Ching-yi Wu, ScD, OTR
}

\begin{abstract}
Background. Selection of patients who are most and least likely to benefit from constraint-induced therapy (CIT) for the upper extremity is uncertain. Objective. This study investigated demographic and clinical characteristics that may predict outcomes for a distributed form of CIT. Methods. A group of 57 patients were treated with distributed CIT, and 7 potential predictors were identified, including age, sex, side of stroke, time since stroke, spasticity, neurologic status, and movement performance of the distal part of the upper extremity. Treatment outcome was assessed in terms of motor performance, perceived functional ability of the affected hand, and functional performance of daily activities, measured by Fugl-Meyer Assessment (FMA), Motor Activity Log (MAL), and Functional Independence Measure (FIM), respectively. Results. Motor ability of the distal part of the upper extremity and time since stroke were significantly predictive of outcomes on the FMA (adjusted $R^{2}$ $=0.18, P=.002$ ) and the MAL subtest quality of movement (adjusted $R^{2}=0.43, P<.0001$ ). Motor ability and age were significant predictors of amount of use measured by the MAL (adjusted $R^{2}=0.20, P=.001$ ). None of the variables exhibited a predictive relationship with the FIM. Conclusions. The best predictor for motor outcomes after distributed CIT was greater motor ability of the distal part of the upper extremity, which is consistent with the presence of residual motor pathways that may respond to training. The FMA may be of value in stratifying patients for their likelihood to benefit from distributed CIT protocols.
\end{abstract}

Keywords: Stroke; Rehabilitation; Constraint-induced therapy; Outcome prediction; Hemiplegia; Upper extremity.

\begin{abstract}
A pproximately $30 \%$ to $66 \%$ of stroke survivors experience persistently impaired upper extremity (UE) movement. ${ }^{1}$ Residual motor deficits in the UE frequently cause disability and permanent dependency on community care. ${ }^{2}$ Constraintinduced therapy (CIT) has been advocated as means to improve motor recovery of the UE and functional use of the affected limb among patients after stroke. Constraint-induced therapy involves restraint of the unaffected UE (eg, during $90 \%$ of the hours the patient is awake per day for 2 weeks), forcing the use of the affected UE (eg, $6 \mathrm{~h} / \mathrm{d}$ on 10 consecutive weekdays), and massed task-related training of the affected UE. ${ }^{3}$ Because the acceptance of CIT among therapists and patients remains poor due to prolonged practice and restraint, ${ }^{4}$ different forms of CIT have been developed. For example, the distributed forms of CIT (distributed CIT) involved 2 to 3 hour training of the affected arm with 6- to 9-hour restraint of the unaffected arm for 2 to 4 weeks..$^{5-11}$ Numerous studies ${ }^{5,6,12-14}$ involving stroke patients have shown that various forms of CIT can enhance motor performance and functional ability of the affected UE. However, possible predictors related to distributed CIT outcome remain unknown. The identification of potential predictors for distributed CIT outcome helps
\end{abstract}

underscore the factors that may affect treatment outcomes and target individuals who benefit the most from the therapy.

Evidence regarding predictors of optimal outcomes in stroke patients after CIT is limited. ${ }^{15-18}$ For example, Fritz et $\mathrm{al}^{15}$ examined the 6 demographic and clinical characteristics of side of stroke, time since stroke, hand dominance, age, sex, and ambulatory status as predictors of functional outcomes of CIT and found that age is the only significant predictor of the amount of use in the affected UE after CIT. Rijntjes et al ${ }^{17}$ also demonstrated that age is related to the quality of movement after CIT. A recent study by Langan and van Donkelaar ${ }^{18}$ found that hand dominance does not influence response to CIT. Other clinical and demographic characteristics of stroke, such as side of stroke, time since stroke, and sex, were not found to be good predictors of CIT outcome.

Nevertheless, Ploughman and Corbett, ${ }^{19}$ using one principle of CIT (restraint of the unaffected hand), mentioned that patients with left hemiplegia appeared to benefit from treatment differently from those with right hemiplegia. In addition, Rijntjes et $\mathrm{al}^{17}$ demonstrated greater improvement in fine motor control after CIT for patients with right hemiplegia than for those with left hemiplegia. Time since stroke and sex are often used or

From the School of Occupational Therapy, College of Medicine, National Taiwan University, and Division of Occupational Therapy, Department of Physical Medicine and Rehabilitation, National Taiwan University Hospital, Taipei (KCL, YWH), and Department of Occupational Therapy and Graduate Institute of Clinical Behavioral Science, Chang Gung University, Taoyuan, Taiwan (CYW); and Department of Occupational Therapy, College of Health and Human Services, California State University, Dominguez Hills, Carson, California (YHH). Address correspondence to Ching-yi Wu, ScD, OTR, Department of Occupational Therapy, Chang Gung University, 259 Wen-hwa 1st Rd, Kwei-shan, Taoyuan, Taiwan. E-mail: cywu@mail.cgu.edu.tw. 
proposed as predictors for recovery or rehabilitation outcomes in stroke patients. ${ }^{20,21}$ These potential predictors warrant further scrutiny in the context of outcome studies of CIT. Fritz et al ${ }^{15}$ suggest that other potential predictors such as neurologic status might be included in outcome research on stroke rehabilitation.

Another work by Fritz et $\mathrm{al}^{16}$ investigated several measures of motor status to predict CIT outcome. They found that active finger extension was the only significant predictor of movement capacity outcome. However, they did not consider indicators of spasticity. Neurophysiologic measures ${ }^{22}$ and the degree of spasticity measured clinically ${ }^{23}$ have also been proposed as predictors for training-related stroke recovery. ${ }^{22,23}$

The studies discussed are not fully comparable, ${ }^{15-18}$ possibly owing to differences in potential predictors, outcome measures, and statistical analyses. Fritz et a ${ }^{15}$ used demographic and clinical characteristics without UE performance, whereas in another study, Fritz et $\mathrm{al}^{16}$ only included motor performance without demographic characteristics. Langan and van Donkelaar ${ }^{18}$ only examined the demographic characteristic of hand dominance. Meanwhile, although Rijntjes et a ${ }^{17}$ comprehensively examined 14 factors possibly influencing CIT outcomes, they performed statistical analysis for each factor without considering their possible interactions or correlations. This study included demographic and clinical characteristics (primarily involving movement performance of the distal part of UE and spasticity) in the predictive model to make it more inclusive and allow study of the possible relations among these factors.

The outcomes of motor performance and functional capacity after CIT used in these studies were primarily assessed using the Wolf Motor Function Test and the Motor Activity Log (MAL). ${ }^{15-18}$ Additional outcome measures of motor and functional performance commonly reported in CIT studies included measurement tools such as the Fugl-Meyer Assessment (FMA) and the Functional Independence Measure (FIM). ${ }^{5,6,12,13,19}$ Whether the outcomes after CIT can be predicted by demographic and clinical characteristics awaits investigation. Furthermore, studied factors that are predictive of positive treatment outcomes are limited to CIT delivery. ${ }^{15,16}$ These factors may not be appropriate predictors of success in distributed CIT delivery, ${ }^{16}$ which is in a less intense manner and for a different duration compared with CIT.

This study aimed to identify predictors of distributed CIT outcomes, including movement performance, perceived functional ability of the affected hand, and functional performance of daily activities measured by FMA, MAL, and FIM. The potential predictors selected in this study were age, sex, side of stroke, time since stroke, spasticity, neurologic status, and the movement performance of the distal part of the upper extremity.

\section{Methods}

\section{Subjects}

A total of 57 patients (39 men, 18 women) poststroke, with a mean age of 55.10 years (range, 14-86 years), were recruited from 2 participating sites from July 2003 to August 2007. The
Table 1

\section{Demographic and Clinical Characteristics} of the 57 Subjects

\begin{tabular}{lc}
\hline Characteristics & \multicolumn{1}{c}{ Value } \\
\hline Age, mean (SD), y & $55.10(13.99)$ \\
Sex, No. (\%) & $18(31.58)$ \\
$\quad$ Female & $39(68.42)$ \\
$\quad$ Male & $12(0.7-88)$ \\
Time since stroke, mean (range), mon & \\
Side of stroke, No. (\%) & $32(56.14)$ \\
$\quad$ Left & $25(43.86)$ \\
$\quad$ Right & \\
Brunnstrom stage of upper extremity & 5 \\
$\quad$ Proximal part, median & 4 \\
$\quad$ Distal part, median & $27(3.08)$ \\
Mini-Mental State Exam, mean (SD) & $0.85(1.01)$ \\
MAL (amount of use), mean (SD) & $0.52(0.47)$ \\
Modified Ashworth Scale, mean (SD) &
\end{tabular}

Abbreviation: MAL, Motor Activity Log.

patients were right-hand dominant before the stroke by selfreport and were a mean of 12 months (range, 0.7-88 months) after onset of a first-ever cerebrovascular accident (Table 1). All patients received independent examinations by a physiatrist and occupational therapist to determine their eligibility for inclusion. The 2 eligibility criteria for participants were that they displayed considerable nonuse of the affected limb, defined as an amount of use (AOU) score of less than 2.5 on the MAL, ${ }^{24}$ and their affected arm had reached Brunnstrom stage 3 or more. ${ }^{25}$

Patients were excluded if they had obvious cognitive impairments (score $<24$ on the Mini-Mental State Examination), ${ }^{26}$ excessive spasticity in the shoulder, elbow, wrist, or finger joints of the affected UE (Modified Ashworth Scale [ASH] score $\geq 2$ in any joint), ${ }^{27}$ and balance problems sufficient to compromise safety when wearing the project's constraint device.

Institutional review board approval was obtained from the study sites, and written informed consent was obtained from each patient before inclusion.

\section{Procedures}

We used a distributed form of CIT involving practice of functional tasks using the affected UE for 2 hours per day 5 days per week and restraint of the unaffected UE for 6 hours per day for 3 weeks. Such a protocol has been shown to be beneficial for motor and functional recovery after stroke $e^{6,9,10}$ and is feasible in clinical settings. Shaping/adaptive and repetitive task practice techniques were administered on an individual basis during the training sessions. The tasks selected by patients and the therapist included opening a drawer, picking up a cup and drinking from it, picking up a phone, and other activities simulating daily life. Approximately $15 \mathrm{~min}$ utes of therapy were spent on normalizing the muscle tone of the affected limb as necessary. During the 3-week period, unaffected hands and wrists of the patients were placed in 
mitts with Velcro straps every weekday for 6 hours identified as a time of frequent arm use.

Before and after the 3-week intervention period, the tests were administered by a blinded rater. Before administration of clinical measures, the blinded rater was trained to properly administer these 2 measures. This training included careful review of written instructions and repeated practice. Rater competence was assessed by a senior certified occupational therapist.

\section{Outcome Measures}

Three main outcome measures were used for this study, including FMA, MAL, and FIM. The FMA, an objective assessment tool, measures motor impairment of stroke patients, whereas the MAL and FIM represent subjective (self-reported) and objective measurements of the functional capacity of stroke patients, respectively.

Fugl-Meyer Assessment. The FMA includes items relevant to movement and coordination of the shoulder, elbow, forearm (proximal arm), and movement of the wrist and hand (distal arm) ${ }^{28,29}$ The maximum total score of the FMA is 66 , and represents optimal performance, and the maximum total scores for the proximal and distal parts are 42 and 24 , respectively. ${ }^{30}$ Tests of movements of the distal parts of the UE include (1) wrist stability and flexion/extension at the elbow flexed at $0^{\circ}$ and $90^{\circ}$, and wrist circumduction; (2) mass flexion and extension of finger; and (3) 5 types of grasping movements. Test-retest reliability, interrater reliability, and construct validity are all well established. ${ }^{30,31}$

Motor Activity Log. The MAL contains 30 structured interview questions related to information about the perceptions regarding the frequency (AOU) and quality of movement (QOM) of the affected arm experienced by patients during their daily lives. ${ }^{24}$ The score of each item for AOU and QOM ranges from 0 to 5 . The reliability and validity of the MAL have been established. ${ }^{32-34}$

Functional Independence Measure. The FIM is a standardized measurement tool with a maximum score of 126 . It comprises 18 items grouped into 6 subscales measuring self-care, sphincter control, transfers, locomotion, communication, and social cognition ability. ${ }^{35}$ Each item is rated from 1 to 7 , with higher score indicating better performance. The FIM has exhibited good interrater reliability. ${ }^{36-40}$ This instrument is appropriate for evaluating interventions that highlight UE rehabilitation after stroke. ${ }^{41}$ Several studies have used the FIM to study functional outcome after stroke rehabilitation., $5,6,10,19,42-44$

\section{Potential Predictors}

Empirical findings and theoretic grounds were used to select the 7 potential predictors of age, sex, side of stroke, time since stroke, motor impairment of the distal part of the UE, spasticity, and neurologic deficit. The scores of the distal arm subsection of the FMA represented motor impairment of the distal part of the UE. ${ }^{29}$ The ASH scale, a 6-point rating where each item is scored from 0 to 5 , was used to evaluate the severity of spasticity. ${ }^{27}$ The National Institutes of Health Stroke Scale (NIHSS) was used to assess the neurologic deficit of stroke patients and is widely used for predicting outcome. ${ }^{45}$ The NIHSS was shown to have good reliability, validity, and ability to predict outcomes. ${ }^{46-49}$

\section{Data Reduction}

The relative change in outcome measure between the pretreatment and posttreatment situations served as the dependent variable. To consider the effect of different starting points, the following formulas were used..$^{50-52}$

The following formula was used to index performance improvements after distributed CIT: [Improved Score (Posttest Pretest)/Max Possible Improvement Score (Total Scores Pretest Scores)] $\times 100 \%$.

In contrast, the following formula was applied when performance deteriorated after distributed CIT: [Improved Score $($ Posttest - Pretest)/Pretest Scores] $\times 100 \%$.

We used these indices to indicate performance improvement and deterioration in this study. This practice was based on the proposal of Marx and Cummings ${ }^{53}$ and has been applied in previous research of outcome prediction after stroke. ${ }^{52}$

\section{Statistical Analysis}

The normality of the data of 4 outcome measures and 7 predictors was statistically verified by the value of skewness $( \pm 1)$ and visually verified using histogram graphs. Only the 2 predictors of time since stroke and the ASH required transformation using the natural $\log [\ln ]$.

Predictors of distributed CIT outcome were identified using 2 steps. First, the Pearson correlation coefficient $(r)$ was used to examine the associations between the predictors at the pretest assessment and the relative change scores on the 4 outcome measures. The selection criterion for the entry of the predictors into the models was set to a value of $P=.25 .^{54} \mathrm{~A}$ probability range of up to .25 was used to avoid the exclusion of important factors in the model development.

In the second step, the remaining predictors (ie, those exhibiting relatively high correlation with outcomes) were used in a backward stepwise procedure to develop a linear regression model for each outcome measure. ${ }^{55}$ Adjusted $R^{2}, P$ values, and regression coefficients $(\beta)$ were thus calculated. In addition, regression diagnostics were performed to verify the basic assumptions. The variance inflation factor was used to examine the presence of multicollinearity among predictors in the models.

\section{Results}

Table 2 reports the mean score of the relative change in each outcome measure and the grouping of subjects according to status of change (improvement, no change, or deterioration). The participants generally improved after distributed CIT, 
Table 2

Mean of the Relative Change Scores and the Grouping of Subjects According to Status of Change

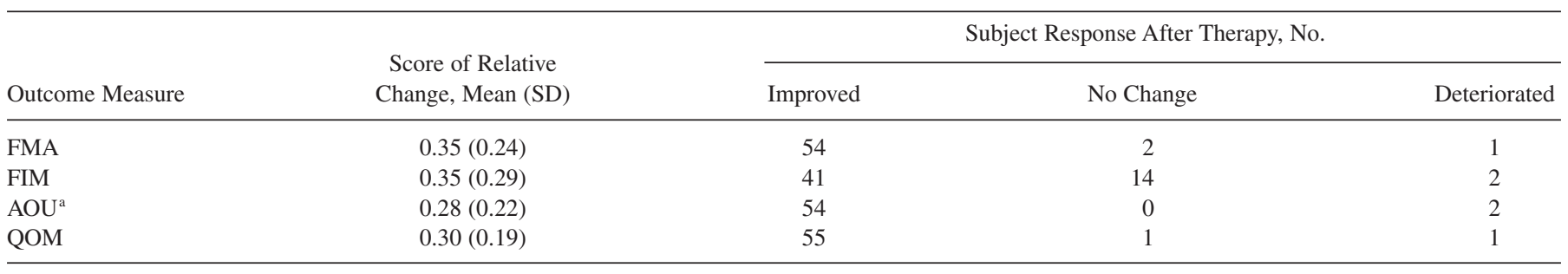

Abbreviations: AOU, amount of use; FIM, Functional Independence Measure; FMA, Fugl-Meyer Assessment; QOM, quality of movement.

${ }^{a}$ One outlier was removed in the AOU model.

especially in measures of motor ability. Only a very small number of subjects deteriorated after therapy.

\section{Multiple Regression Modeling}

Table 3 lists the strength of the associations between the 7 predictors and the relative change scores on the 4 outcome measures. The 4 predictors of [ln] time since stroke, NIHSS, [ln] ASH, and FMA distal part were entered into the FMA model, and the 5 predictors of age, [ln] time since stroke, [ln] ASH, NIHSS, and FMA distal part were entered into both the AOU and QOM models. Finally, the 4 predictors of sex, side of stroke, [ln] time since stroke, and NIHSS were entered into the FIM model.

Table 4 presents the results of backward stepwise multiple regression analyses. The $[\ln ]$ time since stroke and the FMA distal part score were significant predictors for the FMA model, which accounted for $17.7 \%$ of the variance in FMA change scores. The age and FMA distal part score were significant predictors for the AOU model, which accounted for $20.2 \%$ of the variance in AOU change scores. The $[\mathrm{ln}]$ time since stroke and the FMA distal part score were significant predictors for the QOM model, and the model explained $42.8 \%$ of the variance in QOM change scores. No significant predictors were identified for the FIM model. The 3 final regression equations are as follows:

$$
\begin{gathered}
\text { FMA }=(0.346)-(0.071)[\ln ] \text { time since stroke } \\
+(0.010) \mathrm{FMA} \text { distal part } \\
\text { AOU }=(0.301)-(0.004) \text { age }+(0.013) \text { FMA distal part } \\
\text { QOM }=(0.229)-(0.070)[\ln ] \text { time since stroke } \\
+(0.015) \text { FMA distal part }
\end{gathered}
$$

The largest value of the variance inflation factors for the 3 models was 1.14 , indicating that multicollinearity among the predictors did not unduly influence the regression estimates. ${ }^{56}$ This study also examined the appropriateness of the 3 final regression models. Examination of the normal probability plot and the histogram revealed that the residuals were normally distributed. The presence of outliers was examined using Jackknife residual analyses, and residuals exceeding mean \pm 3 $\mathrm{SD}$ were considered outliers. The results demonstrated that the sample contained 1 outlier only in the AOU model. After
Table 3

Relationships Between the 7 Predictors and the Relative Change Scores of the 4 Outcome Measures

\begin{tabular}{lcccc}
\hline & \multicolumn{4}{c}{ Pearson's $r$} \\
\cline { 2 - 5 } Predictors & FMA & FIM & AOU & QOM \\
\hline Age & -0.07 & -0.06 & $-0.25^{\mathrm{a}}$ & $-0.22^{\mathrm{a}}$ \\
Sex & $<0.01$ & $0.17^{\mathrm{a}}$ & $<0.01$ & $<0.01$ \\
Side of stroke & -0.02 & $0.21^{\mathrm{a}}$ & 0.15 & 0.12 \\
[ln] Time since stroke & $-0.38^{\mathrm{a}}$ & $-0.24^{\mathrm{a}}$ & $-0.29^{\mathrm{a}}$ & $-0.47^{\mathrm{a}}$ \\
[ln] ASH & $-0.28^{\mathrm{a}}$ & -0.02 & $-0.22^{\mathrm{a}}$ & $-0.19^{\mathrm{a}}$ \\
NIHSS & $-0.17^{\mathrm{a}}$ & $-0.19^{\mathrm{a}}$ & $-0.37^{\mathrm{a}}$ & $-0.37^{\mathrm{a}}$ \\
FMA distal part & $0.27^{\mathrm{a}}$ & 0.05 & $0.34^{\mathrm{a}}$ & $0.49^{\mathrm{a}}$ \\
\hline
\end{tabular}

Abbreviations: AOU, amount of use; ASH, Modified Ashworth Scale; FMA, Fugl-Meyer Assessment; FIM, Functional Independence Measure; NIHSS, National Institutes of Health Stroke Scale; QOM, quality of movement.

${ }^{\mathrm{a}} P<.25$.

removing the outlier, regression analysis for the AOU model was performed again (Table 4). The regression diagnostic results indicated that the regression analyses were appropriate.

\section{Discussion}

The goal of this study was to investigate 7 demographic and clinical characteristics used to predict motor and functional outcomes after distributed CIT. The analyses revealed that significant predictors varied according to different aspects of outcome after distributed CIT. The best predictor of distributed CIT outcome was motor ability of the distal part of the UE, represented by wrist and hand scores of the FMA. Wrist and hand movement performance could predict changes in motor impairment, amount of use of the affected hand, and quality of movement for daily activities after distributed CIT. The predictive capacity of wrist and hand movement is sensible owing to its important role in the functional use of the UE during daily activities. None of the potential predictors showed a significant predictive relationship with the FIM.

Wrist and hand movement performance and time since stroke were significant variables with a predictive relationship with changes in motor impairment level and quality of movement for performing daily activities after distributed CIT. Better wrist and hand motor ability and shorter duration since 
Table 4

Backward Stepwise Multiple Regression Analyses of the Predictors for the Outcome Measures

\begin{tabular}{|c|c|c|c|c|c|c|}
\hline \multirow{3}{*}{$\begin{array}{l}\text { Adjusted } R^{2} \\
F \text { (significance) }^{2} \\
\text { Predictors }^{\mathrm{a}}\end{array}$} & \multicolumn{2}{|c|}{ FMA } & \multicolumn{2}{|c|}{ AOU } & \multicolumn{2}{|c|}{ QOM } \\
\hline & \multicolumn{2}{|c|}{$\begin{array}{l}0.18 \\
7.03(0.002)\end{array}$} & \multicolumn{2}{|c|}{$\begin{array}{l}0.20 \\
7.98(0.001)\end{array}$} & \multicolumn{2}{|c|}{$\begin{array}{l}0.43 \\
21.96(0.000)\end{array}$} \\
\hline & $\beta$ & $P$ & $\beta$ & $P$ & $\beta$ & $P$ \\
\hline $\begin{array}{l}\text { Age, y } \\
\text { [ln] Time since stroke, mon }\end{array}$ & -0.071 & & -0.004 & .043 & 0.070 & \\
\hline $\begin{array}{l}\text { [n] Time since stroke, mon } \\
\text { FMA distal part }\end{array}$ & $\begin{array}{r}-0.011 \\
0.010\end{array}$ & .040 & 0.013 & .002 & $\begin{array}{r}-0.010 \\
0.015\end{array}$ & .000 \\
\hline Constant & 0.35 & & 0.30 & & 0.23 & \\
\hline
\end{tabular}

Abbreviations: AOU, amount of use; FMA, Fugl-Meyer Assessment; QOM, quality of movement.

${ }^{a}$ The table lists only significant independent variables on the basis of the backward stepwise multiple regression analyses. The table does not list the other variables, including sex, side of stroke, National Institutes of Health Stroke Scale, and [ln] Modified Ashworth Scale, because they were not significantly related to the outcome measures after controlling for the variables in the table. $\beta$ indicates the regression coefficient.

stroke before receiving distributed CIT were associated with reduced impairment level and increased quality of movement after distributed CIT. It might not be surprising that intervention gains accrued to subjects with a higher level of motor control of the distal UE than those with a lower level because the greater the intactness of corticospinal function is, the better the outcome becomes ${ }^{57}$ Shorter time after stroke may be associated with greater potential for recovery, possibly improving response to distributed CIT. It should also be noted that the experience of the subject over that time in practice with the affected UE may be more important than time per se.

These findings are not completely consistent with previous studies, with 1 study ${ }^{16}$ having found only active finger extension predicted the outcome of motor performance after CIT and another ${ }^{15}$ finding no predictors of motor outcome after CIT. The differences among the findings of different models may be explained by the selection of different potential predictors for inclusion in the predictive model and the use of different outcome measures to reflect motor performance.

An implication of this study for further trials of CIT pertains to stratification of patients based on the level of residual hand function. The baseline score of the distal part movements in the FMA, obtained from 12 movements of the wrist and finger, which can be easily and quickly examined, might be used to stratify patients for participation in this form of distributed CIT at this dose. Patients with higher FMA scores may benefit more from this form of CIT than those with lower scores. This stratification based on the initial FMA score differs from the 2-level stratification based on the amount of wrist and digit extension at baseline (ie, dichotomous levels of motor function) in the Extremity Constraint-Induced Therapy Evaluation (EXCITE) trial. ${ }^{14}$ Of note, higher- and lower-functioning patients in the EXCITE trial did not differ significantly in motor function outcomes. ${ }^{14}$ The motor criteria of the EXCITE trial focused on the extent of finger and wrist extension. Our measurements based on the FMA considered additional movements in the wrist and hand, including wrist circumduction and patterns of object grasp. The cumulative scores for the distal movements may have increased values in predicting CIT outcomes.
Wrist and hand performance and age significantly predicted changes in the amount of use of the affected hand after distributed CIT. Patients with enhanced wrist and hand performance and younger age before distributed CIT received greater gains in amount of use of the affected hand from distributed CIT. These findings do not contradict our belief that younger people may preserve better movement capacity and thus exhibit better recovery. ${ }^{58,59}$ Another possibility may be that younger stroke patients require greater abilities to perform daily life tasks and thus even small changes are noted, which was reflected in the self-assessment of AOU score. These results, however, are not entirely consistent with the findings of previous studies, ${ }^{15,17}$ with 1 study ${ }^{15}$ finding age as the only predictor for the outcome of amount of use of the affected hand.

Besides the selection of different predictors for entry into the predictive models or the use of different methods of statistical analysis in these studies, differences in treatment intensity were noted among these studies. Previous studies used a CIT program with more intensive practice (6 hours) of the affected hand and restraint during most waking hours of the unaffected hand each day for 2 weeks, whereas the present study applied distributed CIT according to a less intensive program of 2 hours of practice and 6 hours of restraint per day lasting 3 weeks. The differences between previous studies and the present study suggest that different factors can be used to predict outcomes after CIT or distributed CIT in terms of amount of use in the affected hand.

As described previously, another finding of this study is that none of the potential predictors emerged as significant predictors for the FIM. This lack of association may indicate that the improved motor function did not translate into gains on the FIM because the FIM is a multifaceted scale that involves daily functioning from motor to cognitive domains. In addition, minimal motor improvements may not have been captured in the FIM score because the affected UE may not be used to perform the activities on the FIM (eg, transfers and locomotion). This finding suggests that factors that may affect outcomes in functional independence after distributed CIT differ from those that predicted UE motor improvements. Future research on outcome 
prediction should take additional factors (eg, cognitive and psychosocial functions) into account when the treatment outcomes involve daily function.

Taken together, various significant predictive factors have been identified according to the aspects of outcome to be predicted after distributed CIT. The ideal candidates for obtaining motor gains and subjective functional improvements, as measured by the FMA and the MAL, after distributed CIT might be those that preserve high motor ability of the distal part of UE, who are at a shorter time since stroke, or who are at a younger age. This knowledge should inform patient criteria for treatment success when implementing distributed CIT. For example, patients with higher movement capacity of the UE distal part, in addition to shorter time since stroke, may achieve greater gains from distributed CIT in terms of motor impairment and quality of movement. Higher movement capacity of the UE distal part, together with younger age, may lead to larger improvements in the use of the affected hand after distributed CIT.

Sex, side of stroke, spasticity, and neurologic impairment status were not predictive of any outcome measures examined in this study. These findings were inconsistent with the work of Rijntjes et al, ${ }^{17}$ who found that patients with left stroke exhibited better fine motor control than patients with right stroke. However, Rijntjes et al examined the individual factor, rather than a combination of several factors, to determine its affect on CIT outcomes.

This study did not find evidence to support spasticity as a predictor for distributed CIT. The patients included in this study had mild spasticity ( $<2$ points on the ASH scale), which might have resulted in the failure of this factor to affect treatment outcomes. Neurologic status is believed to be related to stroke recovery, ${ }^{47}$ and patients with higher neurologic status are assumed to possess greater potential to regain motor ability and daily function after treatment. ${ }^{60}$ However, this study suggests that neurologic status before distributed CIT is not a predictor of treatment outcome. Neurologic status may interact with other predictors such as motor ability of the distal part of UE, and time since stroke may be a stronger predictor of treatment outcome than neurologic status. Furthermore, the NIHSS is often used in the acute stroke patients. It might not be sensitive enough to test the neurologic status of chronic patients.

\section{Conclusion}

This study is the first to investigate the potential demographic and clinical characteristics of stroke that can serve to predict distributed CIT outcomes in motor and functional capacity. The findings suggest that different significant predictors were applied to predict various aspects of outcome after distributed CIT. The best predictor for distributed CIT outcome was the motor ability of the distal part of the UE. The initial score of the distal movements in the FMA, obtained from 12 movements of the wrist and fingers, might be used to stratify patients for participation in this form of distributed CIT at this dose. The models developed in this study did not predict a large portion of variance of the outcome measures except for the quality of movement on the MAL. Other potential predictors, such as cognitive, psychosocial, and emotional factors, need to be considered in future research.

\section{Acknowledgements}

This project was supported in part by the National Health Research Institutes (NHRI-EX95-9103EC \& NHRI-EX979742PI) and the National Science Council (NSC-95-2314-B002-225-MY2 and NSC-96-2628-B-002-033-MY2) in Taiwan.

\section{References}

1. Carr J, Shephard R. Neurological Rehabilitation: Optimizing Motor Performance. London: Butterworth-Heineman; 1998.

2. Plummer, AC. Constraint-induced therapy and the motor learning literature that underpins its application. Phys Ther Rev. 2003;8:143-149.

3. Sunderland A, Tuke A. Neuroplasticity, learning and recovery after stroke: a critical evaluation of constraint-induced therapy. Neuropsychol Rehabil. 2005;15:81-96.

4. Page SJ, Levine P, Sisto S, Bond Q, Johnston MV. Stroke patients' and therapists' opinions of constraint-induced movement therapy. Clin Rehabil. 2002;16:55-60.

5. Dromerick AW, Edwards DF, Hahn M. Does the application of constraintinduced movement therapy during acute rehabilitation reduce arm impairment after ischemic stroke? Stroke. 2000;31:2984-2988.

6. Wu CY, Lin KC, Chen HC, Chen IH, Hong WH. Effects of modified constraint-induced movement therapy on movement kinematics and daily function in patients with stroke: a kinematic study of motor control mechanisms. Neurorehabil Neural Repair. 2007;21:460-466.

7. Wolf SL, Winstein CJ, Miller J, Blanton S, Clark P, Nichols-Larsen D. Looking in the rear mirror: the EXCITE trial revisited. Neurorehabil Neural Repair. 2007;21:379-387.

8. Dettmers C, Teske U, Hamzei F, Uswatte G, Taub E, Weiller C. Distributed form of constraint-induced movement therapy improves functional outcome and quality of life after stroke. Arch Phys Med Rehabil. 2005;86:204-209.

9. Lin KC, Wu CY, Wei TH, Lee CY, Liu JS. Effects of modified constraintinduced movement therapy on reach-to-grasp movements and functional performance after chronic stroke: a randomized controlled study. Clin Rehabil. 2007;21:1075-1086.

10. Wu CY, Chen CL, Tsai WC, Lin KC, Chou SH. A randomized, controlled trial of mCIT for elderly stroke survivors: changes in motor impairment, daily functioning, and quality of life. Arch Phys Med Rehabil. 2007;88:273-278.

11. Boake C, Noser EA, Ro T, et al. Constraint-induced movement therapy during early stroke rehabilitation. Neurorehabil Neural Repair. 2007;21:14-24.

12. Bonifer NM, Anderson KM, Arciniegas DB. Constraint-induced movement therapy after stroke: efficacy for patients with minimal upper-extremity motor ability. Arch Phys Med Rehabil. 2005;86:1867-1873.

13. Page SJ, Sisto SA, Levin P, Leonard AC. Modified constraint-induced therapy in acute stroke: a randomized controlled pilot study. Neurorehabil Neural Repair. 2005;19:27-32.

14. Wolf SL, Winstein CJ, Miller JP, et al. Effects of constraint-induced movement therapy on upper extremity function 3 to 9 months after stroke. JAMA. 2006;296:2095-2104.

15. Fritz SL, Light KE, Clifford SN, Patterson TS, Behrman AL, Davis SB. Descriptive characteristics as potential predictors of outcomes following constrain-induced movement therapy for people after stroke. Phys Ther. 2006;86:825-835.

16. Fritz SL, Light KE, Patterson TS, Behrman AL, Davis SB. Active finger extension predicts outcomes after constraint-induced movement therapy for individuals with hemiparesis after stroke. Stroke. 2005;36:1172-1177. 
17. Rijntjes M, Hobbeling V, Hamzei F, et al. Individual factors in constraintinduced movement therapy after stroke. Neurorehabil Neural Repair. 2005; 19:238-239.

18. Langan J, van Donkelaar P. The influence of hand dominance on the response to a constraint-induced therapy program following stroke. Neurorehabil Neural Repair. 2008;22:298-304.

19. Ploughman MP, Corbett D. Can forced-used therapy be clinically applied after stroke? An exploratory randomized controlled trial. Arch Phys Med Rehabil. 2004;85:1417-1423.

20. Dobkin BH. Confounders in rehabilitation trials of task-oriented training: lessons from the designs of he EXCITE and SCILT multicenter trials. Neurorehabil Neural Repair. 2007;21:3-13.

21. Cargano JW, Reeves MJ. Sex differences in stroke recovery and strokespecific quality of life: results from a statewide stroke registry. Stroke. 2007;38:2541-2548.

22. Wittenberg G, Bastings E, Fowlkes A, Morgan T, Good D, Pons T. Dynamic course of intracortical TMS paired-pulse responses during recovery of motor function after stroke. Neurorehabil Neural Repair. 2007;21:568-573.

23. Feys H, De Weerdt W, Nuyens G, van de Winckel A, Selz B, Kiekens C. Predicting motor recovery of the upper limb after stroke rehabilitation: value of a clinical examination. Physiother Res Int. 2000;5:1-18.

24. Wolf SL, Thompson P, Morris DM, et al. The EXCITE trial: Attributes of the Wolf Motor Function Test in patients with subacute stroke. Neurorehabil Neural Repair. 2005;19:194-205.

25. Brunnstrom S. Movement Therapy in Hemiplegia. New York, NY: Harper \& Row; 1970.

26. Folstein MF, Folstein SE, McHugh PR. "Mini-mental State.” A practical method for grading the cognitive state of patients for the clinician. $J$ Psychiatr Res. 1975;12:189-198.

27. Bohannon R, Smith M. Interrater reliability of a modified Ashworth scale of muscle spasticity. Phys Ther. 1987;67:206-207.

28. Fugl-Meyer AR, Jaasko L, Leyman I, Olsson S, Steglind S. The poststroke hemiplegic patient. 1. A method for evaluation of physical performance. Scand J Rehabil Med. 1975;7:13-31.

29. Masiero S, Celi A, Rosati G, Armani M. Robotic-assisted rehabilitation of the upper limb after acute stroke. Arch Phys Med Rehabil. 2007;88:142-149.

30. Duncan PW, Propst M, Nelson SG. Reliability of the Fugl-Meyer assessment of sensorimotor recovery following cerebrovascular accident. Phys Ther. 1983;63:1606-1610.

31. DiFabio RP, Badke RB. Relationship of sensory organization to balance function in patients with hemiplegia. Phys Ther. 1990;70:542-548.

32. van der Lee JH, Beckerman H, Knol DL, de Vet HCW, Bouter LM. Clinimetric properties of the motor activity log for the assessment of arm use in hemiparetic patients. Stroke. 2004;35:1410-1414.

33. Uswatte G, Taub E, Morris D, Light K, Thompson PA. The motor activity $\log$-28: assessing daily use of the hemiparetic arm after stroke. Neurology. 2006;67:1189-1194.

34. Uswatte G, Taub E, Morris D, Vignolo M, McCulloch K. Reliability and validity of the upper-extremity motor activity $\log -14$ for measuring realworld arm use. Stroke. 2005;36:2493-2496.

35. Hamilton BB, Granger CV, Sherwin FS, Zielezny M, Tashman JS. A uniform national data system for medical rehabilitation. In: Fuhrer MJ, ed. Rehabilitation outcomes: analysis and measurements. Baltimore, MD: Brookes; 1987:137-147.

36. Chau N, Daler S, Andre JM, Patris A. Inter-rater agreement of two functional independence scales: the Functional Independence Measure (FIM) and a subjective uniform continuous scale. Disabil Rehabil. 1994;16:63-71.

37. Hamilton B, Laughlin JA, Fiedler RC, Granger CV. Interrater reliability of the 7-level Functional Independence Measure (FIM). Scand J Rehabil Med. 1994;26:115-119.

38. Dodds TA, Martin DP, Stolov WC, Deyo RA. A validation of the Functional Independence Measurement and its performance among rehabilitation inpatients. Arch Phys Med Rehab. 1993;74:531-536.
39. Kidd D, Stewart G, Baldry J, et al. The Functional Independence Measure: a comparative validity and reliability study. Disabil Rehabil. 1995; 17:10-14.

40. Gosman-Hedstrom G, Svensson E. Parallel reliability of the Functional Independence Measure and the Barthel ADL index. Disabil Rehabil. 2000;22:702-715.

41. Wolf SL. Revisiting constraint-induced movement therapy: are we too smitten with the mitten? Is all nonuse "learned"? and other quandaries. Phys Ther. 2007;87:1212-1223.

42. Studenski S, Duncan PW, Perera S, Reker D, Lai SM, Richards L. Daily functioning and quality of life in a randomized controlled trial of therapeutic exercise for subacute stroke survivors. Stroke. 2005;36: 1764-1770.

43. Ergeletzis D, Kevorkian CG, Rintala D. Rehabilitation of the older stroke patient: functional outcome and comparison with younger patients. Am J Phys Med Rehabil. 2002;81:881-889.

44. Shelton FNAP, Volpe BT, Reding M. Motor impairment as a predictor of functional recovery and guide to rehabilitation treatment after stroke. Neurorehabil Neural Repair. 2001;15:229-237.

45. Bruno A, Saha C, Williams LS. Using change in the National Institutes of Health Stroke Scale to measure treatment effect in acute stroke trials. Stroke. 2006;37:920-921.

46. Kasner S, Chalela J, Luciano J, et al. Reliability and validity of estimating the NIH stroke scale score from medical records. Stroke. 1999;30: 1534-1537.

47. Adams HP Jr, Davis PH, Liera EC, et al. Baseline NIH stroke scale score strongly predicts outcome after stroke: a report of the Trial of Org 10172 in acute stroke treatment (TOAST). Neurology. 1999;53:126-131.

48. Lyden P, Lu M, Jackson C, et al. Underlying structure of the National Institutes of Health Stroke Scale: results of a factor analysis. NINDS tPA Stroke Trial Investigators. Stroke. 1999;30:2347-2354.

49. Young FB, Weir CJ, Lees KR. Comparison of the National Institutes of Health Stroke Scale with disability outcome measures in acute stroke trials. Stroke. 2005;36:2187-2192.

50. Heinemann AW, Roth EJ, Cichowski MS, Betts HB. Multivariate analysis of improvement and outcome following stroke rehabilitation. Arch Neurol. 1987;44:1167-1172.

51. Shah S, Vanclay F, Cooper B. Efficiency, effectiveness and duration of stroke rehabilitation. Stroke. 1990;21:241-246.

52. Kugler C, Altenhöner T, Lochner P, Ferbert A. Does age influence early recovery from ischemic stroke? A study from the Hessian stroke data bank. J Neurol. 2003;250:676-681.

53. Marx JD, Cummings K. Physics education research. Am J Phys. 2007;75:87-91.

54. Hosmer DW, Lemeshow S. Applied Logistic Regression. New York, NY: John Wiley \& Sons; 1989.

55. Kleinbaum DG, Kupper LL, Muller KE. Applied Regression Analysis and Other Multivariable Methods. 2nd ed. Boston, MA: PWS-Kent; 1988.

56. Neter J, Kutner M, Nachtsheim C, Wasserman W. Applied Linear Statistical Models. 4th ed. Chicago, IL: McGraw-Hill; 1996.

57. Cho SH, Kim DG, Kim DS, Kim YH, Lee CH, Jang SH. Motor outcome according to the integrity of the corticospinal tract determined by diffusion tensor tractography in the early stage of corona radiata infarct. Neurosci Lett. 2007;426:123-127.

58. Patti F, Failla G, Reggio A, et al. Stroke in the young in Sicily: prevalence and clinical features. J Stroke Cerebrovasc Dis. 1998;7:196-199.

59. Jongbloed L. Prediction of function after stroke: a critical review. Stroke. 1986;17:765-776.

60. Hofgren C, Bjorkdahl A, Esbjornsson E, Sunnerhagen KS. Recovery after stroke: cognition, ADL function and return to work. Acta Neurol Scand. 2007; $115: 73-80$ 